\title{
Measurements of $R^{\mathrm{d}}-R^{\mathrm{p}}$ and $R^{\mathrm{Ca}}-R^{\mathrm{C}}$ in deep inelastic muon scattering
}

\author{
New Muon Collaboration
}

Bielefeld University-CERN-Freiburg University-Max Planck Institüt für Kernphysik, Heidelberg-Heidelberg UniversityMainz University-Mons University-Neuchatel University-NIKHEF-K-Oxford University-Saclay DAPNIA/SPPUniversity of California, Santa Cruz-Paul Scherrer Institute-Torino University and INFN Torino-Uppsala UniversityInstitute for Nuclear Studies, Warsaw-Warsaw University-Wuppertal University

\author{
P. Amaudruz ${ }^{\mathrm{a}, 1}$, M. Arneodo ${ }^{\mathrm{b}}$, A. Arvidson ${ }^{\text {c }}$, B. Badelek ${ }^{\text {d }}$, G. Baum ${ }^{\mathrm{e}}$, J. Beaufays ${ }^{\mathrm{f}, 2}$, \\ I.G. Bird ${ }^{\text {g,3 }}$, M. Botje ${ }^{\text {a, } 4}$, C. Broggini h,5, W. Brückner ${ }^{\text {g }}$, A. Brüll ${ }^{\text {i }}$, W.J. Burger ${ }^{\text {a,6 }}$, \\ J. Ciborowski f,7, R. van Dantzig f, H. Döbbeling ${ }^{\text {g,8 }}$, J. Domingo ${ }^{\text {a,9 }}$, J. Drinkard ${ }^{\text {j, }}$, H. Engelien ${ }^{\text {i, }}$, \\ M.I. Ferrero ${ }^{\text {b }}$, L. Fluri ${ }^{\text {h }}$, P. Grafström ${ }^{\text {c, } 10}$, T. Granier ${ }^{\text {k }}$, D. von Harrach ${ }^{\text {g, } 11}$, \\ M. van der Heijden ${ }^{f}, C$. Heusch ${ }^{j}$, Q. Ingram ${ }^{a}$, K. Janson-Prytz ${ }^{c}$, M. de Jong ${ }^{f, 11}$, \\ E.M. Kabuß ${ }^{\text {g, } 11}$, R. Kaiser ${ }^{\mathrm{i}}$, T.J. Ketel ${ }^{\text {f }}$, F. Klein ${ }^{\ell}$, B. Korzen ${ }^{\text {m }}$, U. Kruner ${ }^{\mathrm{m}}$, S. Kullander ${ }^{\text {c }}$, \\ U. Landgraf $^{\mathrm{i}}$, F. Lettenström ${ }^{\mathrm{j}}$, T. Lindqvist ${ }^{\mathrm{c}}$, G.K. Mallot ${ }^{\mathrm{n}, \ell}$, C. Mariotti $^{\mathrm{b}, 12}$, \\ G. van Middelkoop ${ }^{\text {n,f }}$, A. Milsztajn ${ }^{\text {k, }}$, Y. Mizuno ${ }^{\text {g,13 }}$, J. Nassalski ${ }^{\circ}$, D. Nowotny ${ }^{\mathrm{g}, 14}$, \\ N. Pavel ${ }^{\mathrm{m}, 15}$, C. Peroni ${ }^{\text {b }}$, H. Peschel ${ }^{\mathrm{m}, 16}$, B. Povh ${ }^{\text {g,p }}$, R. Rieger ${ }^{\ell}$, K. Rith ${ }^{\mathrm{g}}$, K. Röhrich ${ }^{\ell, 17}$, \\ E. Rondio ${ }^{d}$, L. Ropelewski d ${ }^{\text {, A. Sandacz }}{ }^{\circ}$, C. Scholz $^{8}$, R. Schumacher ${ }^{\text {a, } 18}$, U. Sennhauser ${ }^{\text {a, } 19}$, \\ F. Sever ${ }^{\mathrm{e}, 20}$, T.-A. Shibata ${ }^{\mathrm{p}}$, M. Siebler ${ }^{\mathrm{e}}$, A. Simon ${ }^{\mathrm{g}}$, A. Staiano ${ }^{\mathrm{b}}$, G. Taylor ${ }^{\mathrm{q}, 21}$, \\ M. Treichel ${ }^{\mathrm{g}, 22}$, M. Virchaux ${ }^{\mathrm{k}}$, J.L. Vuilleumier ${ }^{\mathrm{h}}$, T. Walcher ${ }^{\ell}$, \\ R. Windmolders ${ }^{\mathrm{a}}$ and F. Zetsche ${ }^{\mathrm{g}}$ \\ a Paul Scherrer Institute, CH-5234 Villigen, Switzerland \\ b Università di Torino, Instituto di Fisica, 1-10125 Turin, Italy \\ - Department of Radiation Science, University of Uppsala, S-75121 Uppsala, Sweden \\ d University of Warsaw, PL-00681 Warsaw, Poland \\ - Physics Department, Bielefeld University, W-4800 Bielefeld, $F R G^{23}$ \\ f NIKHEF-K, P.O. Box 4395, NL-1009 AJ Amsterdam, The Netherlands 24 \\ 8 Max Planck Institut für Kernphysik, W-6900 Heidelberg, FRG ${ }^{23}$ \\ h Université de Neuchatel, $\mathrm{CH}-2000$ Neuchatel, Switzerland \\ i Physics Department, Freiburg University, W-7800 Freiburg, FRG ${ }^{23}$ \\ j Institute for Particle Physics, University of California, Santa Cruz, 95064 CA, USA \\ k DAPNIA/SPP, CE Saclay, F-91191 Gif-sur-Yvette, France \\ ${ }^{\ell}$ Institut für Kernphysik, Mainz University, W-6500 Mainz, FRG ${ }^{23}$ \\ m Physics Department, Wuppertal University, W-5600 Wuppertal, $F R G^{23}$ \\ n CERN, CH-1211 Geneva 23, Switzerland \\ - Institute for Nuclear Studies, PL-00681 Warsaw, Poland \\ p Heidelberg University, W-6900 Heidelberg, FRG ${ }^{23}$ \\ a Nuclear Physics Laboratory, University of Oxford, Oxford OX1 3RH, UK
}


Results are presented on the difference in $R$, the ratio of longitudinally to transversely polarised virtual photon absorption cross sections, for the deuteron and the proton. They are obtained by comparing the ratio of cross sections for the deep inelastic scattering of muons from deuterium and hydrogen targets at 90 and $280 \mathrm{GeV}$ incident energy. The results cover the range $x=0.01-$ 0.30 , at an average $Q^{2}$ of $9 \mathrm{GeV}^{2}$. The measured difference $R^{\mathrm{d}}-R^{\mathrm{p}}$ shows no significant $x$ dependence and is compatible with zero, as well as with expectations from perturbative QCD. We use the same method to obtain the difference $R^{\mathrm{Ca}}-R^{\mathrm{C}}$ from cross section ratios measured on carbon and calcium targets at 90 and $200 \mathrm{GeV}$ incident energy.

\section{Introduction}

In a recent article, we presented the ratio of structure functions of the neutron and proton, $F_{2}^{\mathrm{n}}\left(x, Q^{2}\right) / F_{2}^{\mathrm{P}}\left(x, Q^{2}\right)$, obtained from a measurement on hydrogen and deuterium targets simultaneously exposed to the CERN muon beam [1]. This high statistics result was obtained at two settings of

1 Present address:TRIUMF, Vancouver, B.C., Canada V6T 2 A3.

2 Present address: Trasys, Brussels, Belgium.

3 Present address: Saclay, DAPNIA, F-91191 Gif-sur-Yvette, France.

4 Present address: NIKHEF-H, NL-1009 AJ Amsterdam, The Netherlands.

5 Present address: INFN, Laboratori Nazionali del Gran Sasso, I-67010 Assergi, Italy.

${ }^{6}$ Present address: Université de Genève, $\mathrm{CH}-1211$ Geneva, Switzerland.

7 Present address: University of Warsaw, PL-00681 Warsaw, Poland.

${ }^{8}$ Present address: GSI, W-6100 Darmstadt, FRG.

9 Present address: CEBAF, Newport News, VA 23606, USA.

10 Present address: CERN, CH-1211 Geneva 23, Switzerland.

11 Present address: University of Mainz, W-6500 Mainz, FRG.

12 Present address: INFN-Istituto Superiore di Sanita, I-00161 Rome, Italy.

13 Present address: Osaka University, 567 Osaka, Japan.

14 Present address: SAP AG, W-6909 Walldorf, FRG.

15 Present address: DESY, W-2000 Hamburg 52, FRG.

${ }^{16}$ Present address: Gruner und Jahr AG\&CoKG, W-2210 Itzhoe, FRG.

17 Present address: IKP2-KFA, W-5170 Jülich, FRG.

18 Present address: Carnegie Mellon University, Pittsburgh, PA 15213 USA.

19 Present address: EMPA, CH-8600 Dübendorf, Switzerland.

${ }^{20}$ On leave from Jozef Stefan Institut, Ljubljana, Slovenia; present address: NIKHEF-K, NL-1009 AJ Amsterdam, The Netherlands.

${ }^{21}$ Present address: University of Melbourne, Parkville, Victoria, Australie.

22 Present address: Université de Neuchatel, CH-2000 Neuchatel, Switzerland.

${ }^{23}$ Supported by Bundesministerium für Forschung und Technologie.

${ }^{24}$ Supported in part by FOM, Vrije Universiteit Amsterdam and NWO. the mean incident muon energy, 90 and $280 \mathrm{GeV}$, and is characterised by low systematic errors, due to the target arrangement used. The structure function ratio $F_{2}^{\mathrm{n}} / F_{2}$ was derived from the cross section ratio $\sigma^{\mathrm{d}} / \sigma^{\mathrm{p}}$ under the hypothesis that $R$, the ratio of longitudinally to transversely polarised virtual photon absorption cross sections, is the same for the deuteron and the proton. In the present letter we use the same data to check this hypothesis and to measure the difference $R^{\mathrm{d}}-R^{\mathrm{p}}$ by comparing the cross section ratios measured at the two incident muon energies. This method is also applied to the ratio of structure functions measured on calcium and carbon targets at 90 and $200 \mathrm{GeV}$ [2] to obtain the difference $R^{\mathrm{Ca}}-R^{\mathrm{C}}$.

The NM Collaboration target set-up was designed for the determination of cross section ratios with minimal systematic errors. Since such ratios are much more sensitive to $\Delta R$ - the difference in $R$ between the two target materials - than to the individual values of $R$ for each material, these data sets are especially well suited for a measurement of $\Delta R$. The description of the complementary target set-up and all other experimental details of the measurements can be found in refs. [1,2].

Previously, values of $R^{\mathrm{d}}-R^{\mathrm{p}}$ were obtained from a reanalysis of older SLAC data [3] and from measurements of $R^{\mathrm{d}}$ and $R^{\mathrm{p}}$ by the BCDMS Collaboration [4]. In both experiments, the hydrogen and deuterium targets were not exposed simultaneously to the beam in contrast to the conditions of the present experiment. Experimental results on $R^{\mathrm{d}}-R^{\mathrm{p}}$ can be compared to perturbative QCD computations [5]. that predict the difference $\Delta R$ to be much smaller than $R$ if similar gluon distributions in the proton and deuteron are assumed. Thus, large values for $\Delta R$ would give an indication either of a difference in the gluon distribution of the deuteron and the proton, or of different higher-twist contributions to $R^{\mathrm{d}}$ and $R^{\mathrm{p}}$. The same conclusion holds for any pair of target nuclei. 


\section{Principle of the measurement}

In the one-photon exchange approximation, the cross section per nucleon for the deep inelastic scattering of muons off a target nucleus $A$ is related to the structure function per nucleon $F_{2}^{\mathrm{A}}$ and to $R^{\mathrm{A}}$ through the relation

$$
\begin{aligned}
& \frac{\mathrm{d}^{2} \sigma^{\mathrm{A}}}{\mathrm{d} x \mathrm{~d} Q^{2}}=\frac{4 \pi \alpha^{2} F_{2}^{\mathrm{A}}\left(x, Q^{2}\right)}{Q^{4} x} \\
& \quad \times\left(1-y-\frac{Q^{2}}{4 E^{2}}+\frac{y^{2}+Q^{2} / E^{2}}{2\left[1+R^{\mathrm{A}}\left(x, Q^{2}\right)\right]}\right),
\end{aligned}
$$

where $E$ is the incoming muon energy, $-Q^{2}$ the square of the four-momentum transfer, $x=Q^{2} / 2 M \nu$ the Bjorken scaling variable, $\nu$ the energy transfer, $y=\nu / E$ and $M$ the proton mass. To obtain this cross section, the measured total cross section has to be corrected for higher-order QED effects, as discussed for example in ref. [1].

Let the one-photon exchange cross section of eq. (1) be given for different data sets, and let $E_{i}$ be the mean incident muon energy for the $i$ th data set. From eq. (1) it then follows that, for any pair of target nuclei $A$ and $B$, the cross section ratio $\sigma^{\mathrm{B}} / \sigma^{\mathrm{A}}$ for the $i$ th data set at a given $\left(x, Q^{2}\right)$ is a function of three quantities, the structure function ratio $F_{2}^{\mathrm{B}} / F_{2}^{\mathrm{A}}, R^{\mathrm{A}}$ and $R^{\mathrm{B}}$, through

$\frac{\sigma^{\mathrm{B}}}{\sigma^{\mathrm{A}}}\left(E_{i}\right)=\frac{F_{2}^{\mathrm{B}}}{F_{2}^{\mathrm{A}}} \frac{1+R^{\mathrm{A}}}{1+R^{\mathrm{B}}} \frac{1+z_{i} R^{\mathrm{B}}}{1+z_{i} R^{\mathrm{A}}}$.

In eq. (2), the $x$ and $Q^{2}$ dependences have been omitted for clarity. The dependence of the cross section ratio on $E_{i}$ appears only through the kinematic coefficients $z_{i}$,

$z_{i}=\frac{1}{1+\frac{1}{2}\left(y_{i}^{2}+Q^{2} / E_{i}^{2}\right) /\left(1-y_{i}-Q^{2} / 4 E_{i}^{2}\right)}$,

where $y_{i}=\nu / E_{i}$. The $z_{i}$ coefficients are always smaller than unity, and mostly a function of $y_{i}$ only.

If $R^{\mathrm{B}}$ and $R^{\mathrm{A}}$ are equal, the cross section ratio in eq. (2) does not depend on $R$. Expanding eq. (2) to first order in $\Delta R=R^{\mathrm{B}}-R^{\mathrm{A}}$, one obtains

$\frac{\sigma^{\mathrm{B}}}{\sigma^{\mathrm{A}}}\left(E_{i}\right)=\frac{F_{2}^{\mathrm{B}}}{F_{2}^{\mathrm{A}}}\left(1-\frac{1-z_{i}}{(1+\bar{R})\left(1+z_{i} \bar{R}\right)} \Delta R+\ldots\right)$,

where $\bar{R}=\frac{1}{2}\left(R^{\mathrm{A}}+R^{\mathrm{B}}\right)$. The dependence of the cross section ratio on $E_{i}$ is weak: for an arbitrary value $\Delta R=0.02$ the variation of this ratio with $E_{i}$ does not exceed one percent for the present experiment. This shows the importance of a measurement with small systematic and statistical errors. As can be from eq. (4), such a variation is more pronounced for values of $\left(x, Q^{2}\right)$ where the difference in $z_{i}$ coefficients is large. If one of the $z^{i}$ 's is close to zero (i.e. $y_{i}$ is large), the variation is largest. This variations is also much more sensitive to $\Delta R$ than to the value of $\bar{R}$.

In the present analysis, data from only two incident muon energies are available. Consequently one cannot measure, in any single $\left(x, Q^{2}\right)$ cell, the three quantities $F_{2}^{\mathrm{B}} / F_{2}^{\mathrm{A}}, R^{\mathrm{A}}$ and $R^{\mathrm{B}}$. In this cell, one can however derive a constraint on $\Delta R$ and $\vec{R}$ that is independent of the structure function ratio, simply by computing the ratio of the cross section ratios from the two data sets. This constraint is visualized in fig. 1 for the case of the proton and the deuteron for one of the $\left(x, Q^{2}\right)$ cells where the cross section ratio is most sensitive to $\Delta R$. In this figure, the solid line is the constraint in the $(\Delta R, \bar{R})$ plane and the dashed lines represent the one standard deviation statistical error on this constraint. Fig. 1 illustrates the different sensitivity of the measured cross section ratios to $\Delta R$ and $\bar{R}$ in a single $\left(x, Q^{2}\right)$ cell: given only a rough estimate of $\bar{R}$, one can measure $\Delta R$ in this cell.

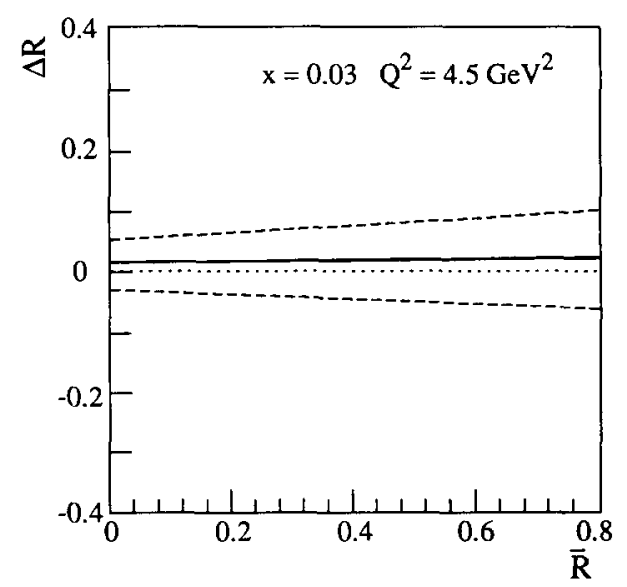

Fig. 1. Constraint obtained in the $(\Delta R, \bar{R})$ plane at $x=0.03$ and $Q^{2}=4.5 \mathrm{GeV}^{2}$ for $\Delta R=R^{\mathrm{d}}-R^{p}$. The solid line visualises the constraint and the dashed lines represent the one standard deviation statistical error on this constraint. This kinematic point, corresponding to $z_{1}=0.20$ and $z_{2}=0.94$, is one of the most sensitive to $\Delta R$ in our data. 
The method so far discussed, whilst independent of the structure function ratio, does not make full use of the measured data, in particular of the cells where only one cross section ratio is available. The data in these cells can be used to constrain the structure function ratio, which depends only weakly on $Q^{2}$, thus improving the measurement of $\Delta R$ from other cells. For this reason, we choose to determine, in each $x$ bin separately, a $Q^{2}$-averaged value of $\Delta R$ in the following way. For all $\left(x, Q^{2}\right)$ cells in a given $x$-bin, we fit to the measured cross section ratios a parametrisation according to eq. (2), with five free parameters: the mean value of $\Delta R$ and of $\bar{R}$ in this $x$-bin, and a 3 parameter function describing the $Q^{2}$ dependence of the structure function ratio in this $x$-bin $F_{2}^{\mathrm{B}} / F_{2}^{\mathrm{A}}=$ $\left(a_{1}+a_{2} \ln Q^{2}\right)\left(1+a_{3} / Q^{2}\right)$. It can be seen - see e.g. ref. [1] - that this form gives a good description of the small $Q^{2}$ dependence of the ratio in any $x$-bin. Because the accuracy with which $\Delta R$ is determined depends slightly on the value of $\bar{R}$ (see fig. 1), we also include in these fits results of previous measurements of $R[6,4]$, obtained in $\left(x, Q^{2}\right)$ domains comparable to that of the present measurement, to loosely constrain the central value of $\bar{R}$. In order to obtain a conservative estimate of the uncertainty on $\Delta R$, we have artificially enlarged in the fits the errors on these other measurements by a factor two; we have checked that using larger error scaling factors leads to very similar results.

\section{Results for the deuteron and the proton}

The results of the fits are shown in fig. 2 in the $x$ range from 0.01 to 0.30 for the data taken with hydrogen and deuterium targets. In the analysis, data at lower $x$ were excluded because there exists no overlap in $Q^{2}$ between the two data sets. Data at larger $x$ were not included because of their low sensitivity to $\Delta R$. In fig. 2 , in each $x$-bin, the two solid lines represent the fitted cross section ratio, which depends on the energy $E_{i}$ through eqs. (2) and (3). In the same figure, the dotted lines represent the (energy-independent) structure function ratio $F_{2}^{\mathrm{B}} / F_{2}^{\mathrm{A}}$ resulting from the same fit; these are shown to indicate the actual effect of a non-zero value of $\Delta R$. The fits describe well the data in all $x$-bins, with a total $\chi^{2} /$ d.o.f. $=$ $143.1 / 128$.
The sources of systematic uncertainty on $\Delta R$ considered here (vertex smearing, normalisation, radiative corrections and momentum calibration ) are further described in ref. [1]. For each individual source separately, the contribution to the uncertainty on $\Delta R$ was computed by modifying the measured cross section ratios by their estimated systematic errors and repeating the fits. All contributions were then added in quadrature to obtain the total systematic error on $\Delta R$. The dominant source of error is the uncertainty on the correction for vertex smearing; the uncertainty on radiative corrections is also important at small $x$. It was checked that imposing $Q^{2}$ cuts up to 1 $\mathrm{GeV}^{2}$ on the data did not change the results.

The values and errors of $\Delta R$ are shown in fig. 3a and listed in table 1 . Because of the rather low systematic errors, the present measurement is limited by the statistical accuracy of the data. No significant $x$ dependence of $\Delta R$ is observed. Averaging the measurements over $x$, one obtains

$\Delta R=R^{\mathrm{d}}-R^{\mathrm{p}}=0.031 \pm 0.016$ (stat.) \pm 0.011 (syst.) , at a mean $Q^{2}$ of $9 \mathrm{GeV}^{2}$. This result is compatible with zero.

Also shown in fig. 3 are the results on $\Delta R$ at higher $x(x \geqslant 0.10)$ obtained from a recent reanalysis of the SLAC data [3] and from the measurements of $R$ by the BCDMS experiment [4]. Both results were obtained from non-simultaneous measurements on hydrogen and deuterium. The agreement is good in both cases and the precision of the SLAC result is comparable to that of the present one. The SLAC and

Table 1

Values of $R^{\mathrm{d}}-R^{\mathrm{p}}$. For each $x$-bin, two mean values of $Q^{2}$ are given, $\left\langle Q^{2}\right\rangle_{\sigma}$ for the cross section ratio data [1] and $\left\langle Q^{2}\right\rangle_{R}$ for the present measurement of $\Delta R$ : the two mean values differ because the data points most sensitive to $\Delta R$ are at high $Q^{2}$ in each $x$-bin.

\begin{tabular}{lccccc}
\hline$x$ & $\begin{array}{l}\left\langle Q^{2}\right\rangle_{\sigma} \\
\left(\mathrm{GeV}^{2}\right)\end{array}$ & $\begin{array}{l}\left\langle Q^{2}\right\rangle_{R} \\
\left(\mathrm{GeV}^{2}\right)\end{array}$ & $R^{\mathrm{d}}-R^{\mathrm{p}}$ & $\begin{array}{l}\text { Stat. } \\
\text { error }\end{array}$ & $\begin{array}{l}\text { Syst. } \\
\text { error }\end{array}$ \\
\hline 0.015 & 2.6 & 3.9 & 0.016 & 0.028 & 0.009 \\
0.030 & 4.2 & 8.2 & 0.034 & 0.028 & 0.009 \\
0.050 & 5.9 & 12.0 & 0.013 & 0.047 & 0.010 \\
0.080 & 7.7 & 22.0 & 0.001 & 0.050 & 0.011 \\
0.125 & 10.0 & 29.5 & 0.139 & 0.075 & 0.011 \\
0.175 & 12.3 & 41.0 & 0.070 & 0.093 & 0.012 \\
0.250 & 15.2 & 66.0 & 0.097 & 0.082 & 0.010 \\
\hline
\end{tabular}




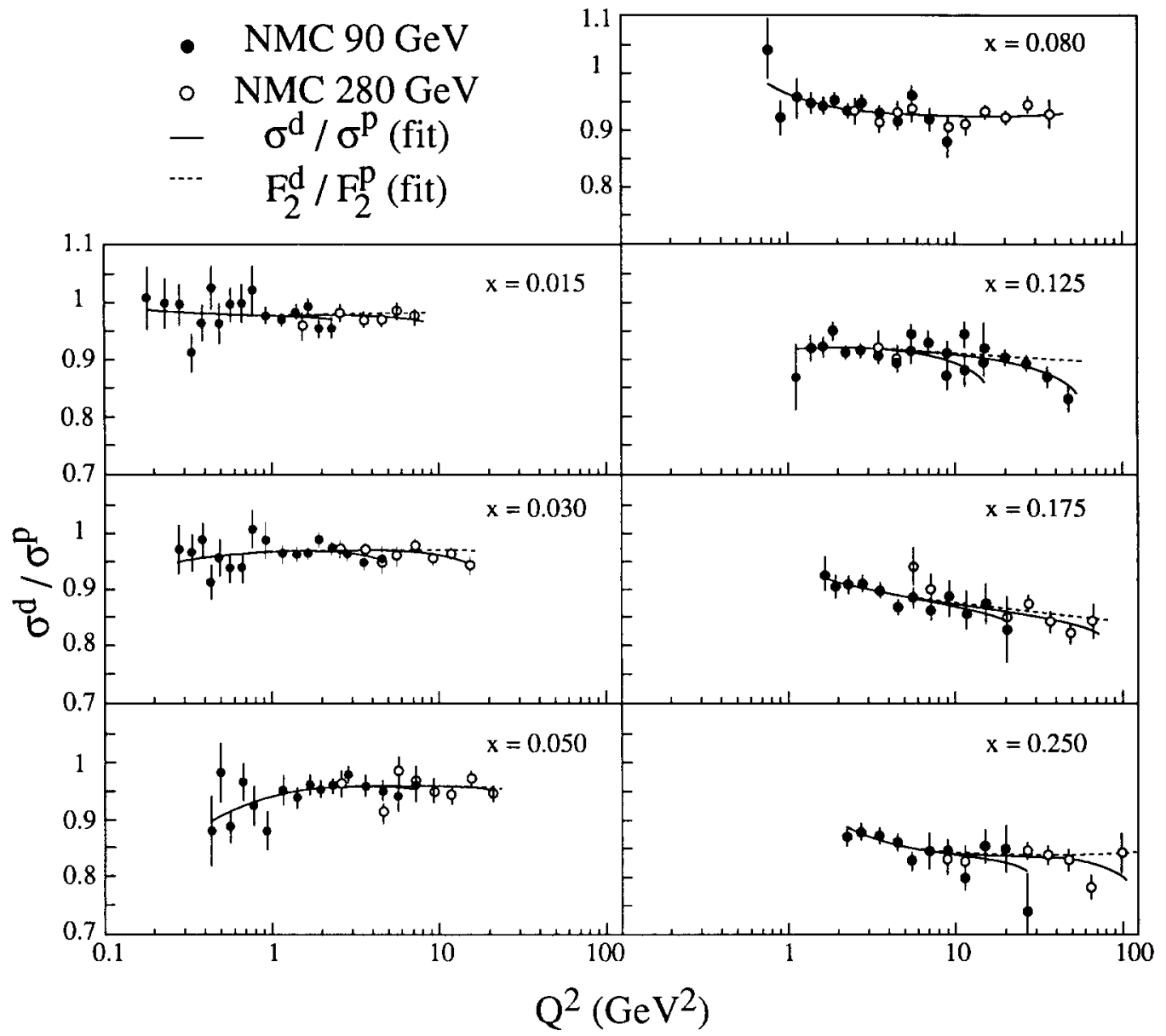

Fig. 2. The measured cross section ratios $\sigma^{\mathrm{d}} / \sigma^{\mathrm{p}}$ as a function of $Q^{2}$, together with the fits described in the text, for seven bins of $x$ between 0.01 and 0.30 . The full (open) symbols correspond to the $90(280) \mathrm{GeV}$ data. The errors shown are statistical only. In each $x$-bin, the two solid lines are the result of the fit. To display the effect of $\Delta R$ on the cross section ratios, the structure function ratio resulting from the same fit is also shown, as a dotted line.

BCDMS data correspond to mean $Q^{2}$ values of about 5 and $35 \mathrm{GeV}^{2}$, respectively.

According to perturbative QCD, the $x$ and $Q^{2}$ dependence of $R$ is related [5] to that of $F_{2}$ and of $x G$, the gluon momentum distribution in the nucleon, through

$R\left(x, Q^{2}\right)=\frac{F_{\mathrm{L}}\left(x, Q^{2}\right)+\left(4 M^{2} x^{2} / Q^{2}\right) F_{2}\left(x, Q^{2}\right)}{F_{2}\left(x, Q^{2}\right)-F_{\mathrm{L}}\left(x, Q^{2}\right)}$,

with

$$
\begin{aligned}
& F_{\mathrm{L}}\left(x, Q^{2}\right)=\frac{\alpha_{\mathrm{s}}\left(Q^{2}\right)}{2 \pi} x^{2} \\
& \quad \times \int_{x}^{1} \frac{\mathrm{d} w}{w^{3}}\left[\frac{8}{3} F_{2}\left(w, Q^{2}\right)+\frac{40}{9}(1-x / w) w G\left(w, Q^{2}\right)\right] .
\end{aligned}
$$

From eqs. (5) and (6), one can compute the difference $\Delta R$ once $F_{2}$ and $x G$ are given. In evaluating $F_{\mathrm{L}}$, we use the $x$-dependence of $F_{2}$ for the proton and the deuteron, including our recent measurement at low $x$ [7], and the gluon distribution obtained from a QCD fit to our data [8]. We obtain a difference $\Delta R$ much 


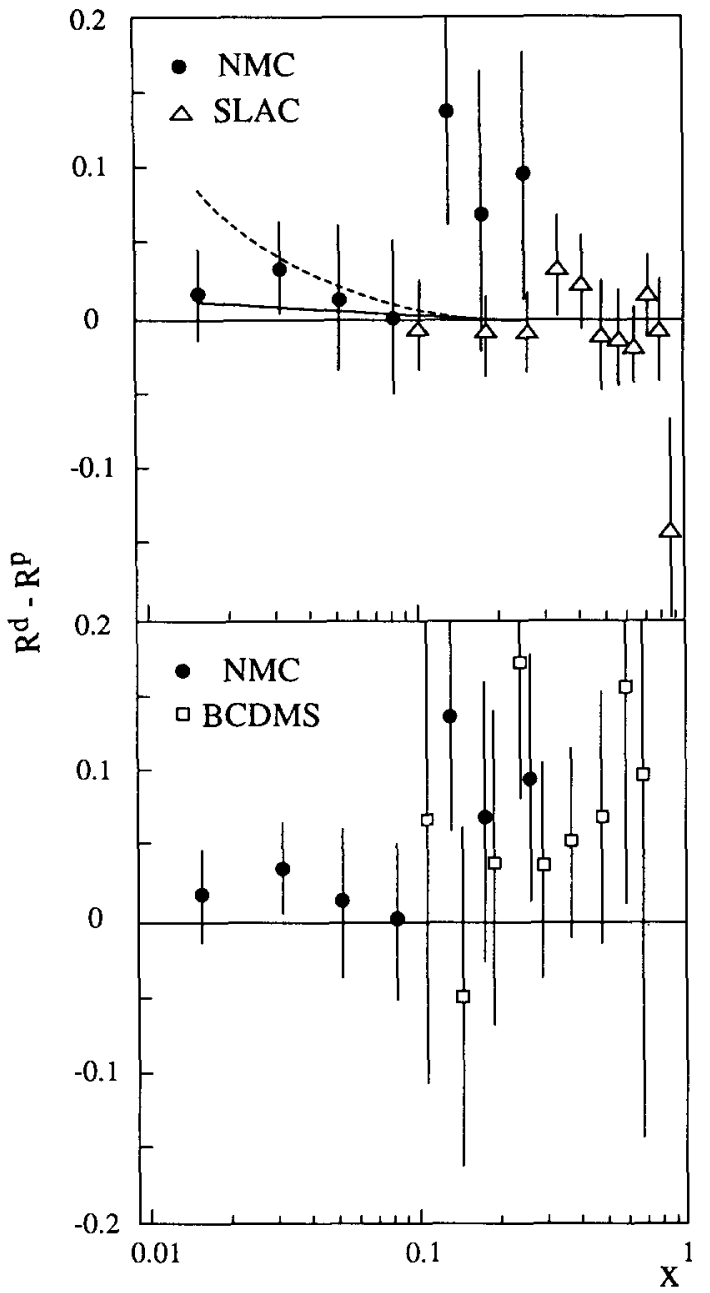

Fig. 3. The measured values of $R^{\mathrm{d}}-R^{\mathrm{p}}$ as a function of $x$. (a) Results from the present analysis. The error bars correspond to statistical and systematic errors added in quadrature. The systematic errors are much smaller than the statistical ones. Also shown are the results from a reanalysis of SLAC electron scattering data [3]. The solid line is the QCD prediction discussed in the text. The dashed line corresponds to the $\mathrm{QCD}$ prediction with the gluon distribution in the deuteron taken to be $10 \%$ larger than that of the proton. (b) The present result compared to that of the BCDMS Collaboration [4].

smaller than $R$, assuming identical gluon distributions in the deuteron and the proton. This QCD prediction for $\Delta R$ is shown in fig. $3 a$ as the solid line; it is in good agreement with our results. If the gluon distributions in the proton and the deuteron are different, eq. (6) leads to predictions for $\Delta R$ that can be much larger at low $x$. As an example, the dashed line in fig. 3a corresponds to the case of gluon distributions in the proton and the deuteron that differ by $10 \%$. Such a difference is close to the uncertainty in the gluon distribution [8]. At the lowest $x(0.015)$, such a large value of $\Delta R$ is not favoured by the present analysis. This indicates that the gluon distributions in the proton and the deuteron do not differ at low $x$ by more than $10 \%$. A similar conclusion was reached in the comparison of $\mathrm{J} / \Psi$ production cross sections on hydrogen and deuterium [9].

\section{Results for calcium and carbon}

Using the same method, we also determined $R^{\mathrm{Ca}}-R^{\mathrm{C}}$ from cross section ratios measured on calcium and carbon targets at 90 and $200 \mathrm{GeV}$ mean incident muon energy [2]. The cross section ratio $\sigma^{\mathrm{Ca}} / \sigma^{\mathrm{C}}$ at $90 \mathrm{GeV}$ was obtained from a simultaneous measurement with a complementary target set-up. In contrast, the cross section ratio at $200 \mathrm{GeV}$ was obtained as the ratio of $\sigma^{\mathrm{Ca}} / \sigma^{\mathrm{D}}$ and $\sigma^{\mathrm{C}} / \sigma^{\mathrm{D}}$, where each of these ratios was obtained from a simultaneous measurement with a complementary target set-up. Because the data sets at 90 and $200 \mathrm{GeV}$ were taken under different experimental conditions, the relative normalisation uncertainty is larger for this measurement than for that of $R^{\mathrm{d}}-R^{\mathrm{p}}$.

The values of $R^{\mathrm{Ca}}-R^{\mathrm{C}}$ resulting from the fits are shown in fig. 4 and listed in table 2 . They cover the range $0.007<x<0.20$. Also here the $x$-range was restricted by the requirement that both data sets overlap in $Q^{2}$ and be sufficiently sensitive to $\Delta R$. The fits describe the data fairly well in all $x$-bins, with a total $\chi^{2} /$ d.o.f. $=304 / 189$. The largest source of systematic error in this measurement is by far the uncertainty on the relative normalisation of the 90 and $200 \mathrm{GeV}$ data sets, which amounts to $0.65 \%$. Since no significant $x$ dependence of $\Delta R$ is observed, we averaged the measurements over $x$ and obtained

$$
\begin{aligned}
\Delta R & =R^{\mathrm{Ca}}-R^{\mathrm{C}} \\
& =0.027 \pm 0.026 \text { (stat.) } \pm 0.020 \text { (syst..), }
\end{aligned}
$$

at a mean $Q^{2}$ of $4 \mathrm{GeV}^{2}$, a result compatible with zero.

Also shown in fig. 4 is the value of $R^{\mathrm{Au}}-R^{\mathrm{Fe}} \mathrm{de}-$ duced from the measurements of $R^{\mathrm{Au}}-R^{\mathrm{D}}$ and $R^{\mathrm{Fe}}-R^{\mathrm{D}}$ in the E140 experiment at SLAC [10], ob- 


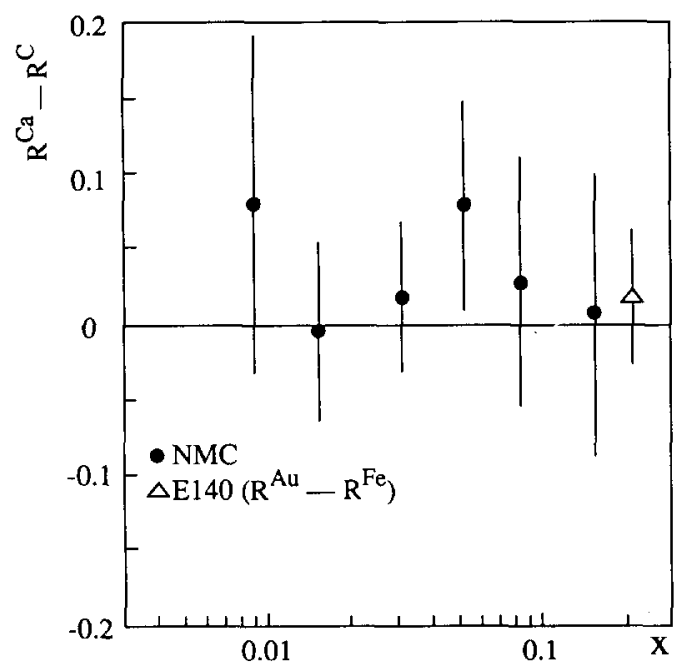

Fig. 4. The measured values of $R^{\mathrm{Ca}}-R^{\mathrm{C}}$ as a function of $x$. The error bars correspond to statistical and systematic errors added in quadrature. Also shown is a value of $R^{\mathrm{Au}}-R^{\mathrm{Fe}}$ deduced from the results of the E140 experiment [10].

tained at a mean $Q^{2}$ of $1.0 \mathrm{GeV}^{2}$. This result is compatible with our data and has a comparable precision.

Table 2

Same as table 1 , for the values of $R^{\mathrm{Ca}}-R^{\mathrm{C}}$. The systematic error corresponds to the normalisation uncertainty only.

\begin{tabular}{lllrll}
\hline$x$ & $\begin{array}{l}\left\langle Q^{2}\right\rangle_{\sigma} \\
\left(\mathrm{GeV}^{2}\right)\end{array}$ & $\begin{array}{l}\left\langle Q^{2}\right\rangle_{R} \\
\left(\mathrm{GeV}^{2}\right)\end{array}$ & $R^{\mathrm{Ca}}-R^{\mathrm{C}}$ & $\begin{array}{l}\text { Stat. } \\
\text { error }\end{array}$ & $\begin{array}{l}\text { Syst. } \\
\text { error }\end{array}$ \\
\hline 0.0085 & 0.9 & 1.0 & 0.080 & 0.108 & 0.024 \\
0.015 & 1.4 & 1.8 & -0.004 & 0.056 & 0.020 \\
0.030 & 2.1 & 3.6 & 0.019 & 0.044 & 0.020 \\
0.050 & 2.6 & 6.0 & 0.080 & 0.065 & 0.020 \\
0.080 & 3.3 & 9.0 & 0.029 & 0.080 & 0.023 \\
0.150 & 5.3 & 15. & 0.009 & 0.089 & 0.025 \\
\hline
\end{tabular}

\section{Summary}

We have presented a determination of $R^{\mathrm{d}}-R^{\mathrm{p}}$, obtained from a simultaneous measurement of the deep inelastic cross sections on hydrogen and deuterium targets. This is the first measurement of such a quantity at $x<0.10$, where the individual values of $R$ for hydrogen and deuterium are expected to be large. The results show no $x$-dependence and are compatible with zero, as well as with the predictions of perturbative QCD, within rather small errors. This can be considered as evidence for the similarity of the gluon distributions in the proton and the deuteron. In addition, we have determined $R^{\mathrm{Ca}}-R^{\mathrm{C}}$, which we also find to be compatible with zero.

\section{References}

[1] NM Collab., P. Amaudruz et al., Nucl. Phys. B 371 (1992) 3.

.[2] NM Collab., P. Amaudruz et al., Z. Phys. C 51 (1991) 387; C 53 (1992) 73.

[3] L.W. Whitlow et al., Phys. Lett. B 250 (1990) 193.

[4] BCDMS Collab. A.C. Benvenuti et al., Phys. Lett. B 223 (1989) 485; B 237 (1990) 592.

[5] G. Altarelli and G. Martinelli, Phys. Lett. B 76 (1978) 89.

[6] CDHSW Collab., P. Berge et al., Z. Phys. C 49 (1991) 187.

[7] NM Collab., P. Amaudruz et al., preprint CERN-PPE/92124, submitted to Phys. Lett. B.

[8] NM Collab., M. Botje, preprint NIKHEF H +K 92-01, in: Proc. 27th Moriond Meeting on QCD and high energy hadronic interactions (Les Arcs, 1992) (Editions Frontières, Gif-sur-Yvette), to appear.

[9] NM Collab., D. Allasia et al., Phys. Lett. B 258 (1991) 493. [10] S. Dasu et al., Phys. Rev. Lett. 60 (1988) 2591. 